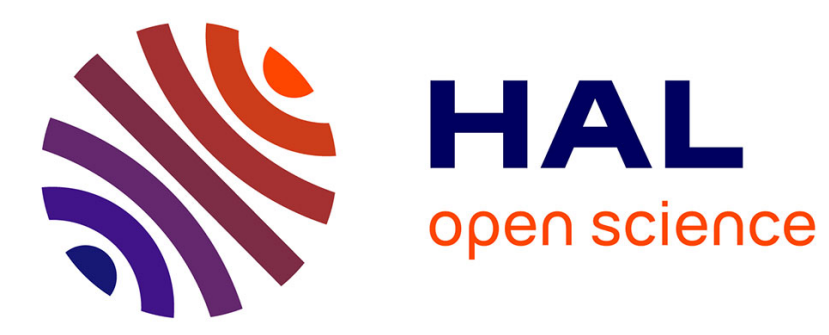

\title{
Visual Servoing-Based approach for efficient autofocusing in Scanning Electron Microscope.
}

Naresh Marturi, Brahim Tamadazte, Sounkalo Dembélé, Nadine Piat

\section{To cite this version:}

Naresh Marturi, Brahim Tamadazte, Sounkalo Dembélé, Nadine Piat. Visual Servoing-Based approach for efficient autofocusing in Scanning Electron Microscope.. IEEE/RSJ International Conference on Intelligent Robots and Systems, IROS'13., Nov 2013, Tokyo, Japan. pp.2677-2682. hal00876197

\section{HAL Id: hal-00876197 \\ https://hal.science/hal-00876197}

Submitted on 24 Oct 2013

HAL is a multi-disciplinary open access archive for the deposit and dissemination of scientific research documents, whether they are published or not. The documents may come from teaching and research institutions in France or abroad, or from public or private research centers.
L'archive ouverte pluridisciplinaire HAL, est destinée au dépôt et à la diffusion de documents scientifiques de niveau recherche, publiés ou non, émanant des établissements d'enseignement et de recherche français ou étrangers, des laboratoires publics ou privés. 


\title{
Visual Servoing-Based Approach for Efficient Autofocusing in Scanning Electron Microscope
}

\author{
Naresh Marturi, Brahim Tamadazte, Sounkalo Dembélé, and Nadine Piat
}

\begin{abstract}
Fast and reliable autofocusing methods are essential for performing automatic nano-objects positioning tasks using a scanning electron microscope (SEM). So far in the literature, various autofocusing algorithms have been proposed utilizing a sharpness measure to compute the best focus. Most of them are based on iterative search approaches; applying the sharpness function over the total range of focus to find an image in-focus. In this paper, a new, fast and direct method of autofocusing has been presented based on the idea of traditional visual servoing to control the focus step using an adaptive gain. The visual control law is validated using a normalized variance sharpness function. The obtained experimental results demonstrate the performance of the proposed autofocusing method in terms of accuracy, speed and robustness.
\end{abstract}

\section{INTRODUCTION}

Over the past few decades, micro-nanosystems have received much attention due to their significant potential for development and use in different industrial domains (telecoms, automotive, aeronautics) and biomedical applications (manipulation of oocytes, non-invasive surgery, nanomedicine). The consequence of this strong demand is the emergence of an active research area concerning the development of assembly and handling technologies on a micro-nanoscale. In the case of nanoelectromechanical systems (NEMS) manufacturing, it is essential to master positioning, transfer, handling or assembling nanometric objects. To be able to visualize these type of objects, it is necessary to use a specific imaging system, the SEM. Compared to the optical microscopes, a SEM produce images of a sample by raster scanning the sample surface with a focused beam of electrons. Therefore, it is characterized by high resolution (better than 1 nanometer), high magnification (up to 500,000×) and a large depth-of-field.

In general, the automation of nanohandling tasks inside a SEM vacuum chamber with nanometric accuracy requires, the use of SEM vision feedback in control laws [1]. Such control strategies require efficient image processing algorithms to extract visual features in designing the control laws. Hence, it is essential to use high quality and sharp images to ensure nanometric precision when extracting visual features. To obtain a sharp image and to use the SEM at its maximum

This work is conducted with a financial support from the project NANOROBUST (ANR- 11-NANO-006) funded by the Agence Nationale de la Recherche (ANR), France. It is also performed in the framework of the Labex ACTION (ANR-11-LABX-01-01) and the Equipex ROBOTEX (ANR-10-EQPX-44-01) projects. It is also partially supported by UFC-BQR project.

Naresh Marturi, Brahim Tamadazte, Sounkalo Dembélé, and Nadine Piat are with Automatic control and Micro Mechatronic Systems (AS2M) department, Institute FEMTO-ST, Besançon, France naresh . marturi at femto-st.fr potential, it is necessary to apply an efficient, accurate, robust and fast autofocus before nanohandling processes.

Basically in vision systems, focusing is defined as a process of obtaining maximum sharpness in the images by adjusting the objective lens. In general, autofocusing methods are classified into two types: active and passive [2]. At first hand, active methods are commonly seen in the modern day cameras where the system uses a different measurement system to find the distance between the object and lens. Autofocusing is achieved by adjusting the optical system. On the other hand, passive methods determine the best focus by analyzing the image recorded at the sensor. The developed autofocusing technique in this work is a passive method that uses SEM secondary electron images.

Traditional autofocusing methods are mostly concentrated on selecting an optimal sharpness function and applying it over total range of focus steps to find the maximum sharpness point (i.e. maximum focus score). A comparison of different sharpness functions has been performed in [3], [4]. For SEM, with a high range of focus, applying the sharpness function at each focus step and finding the sharp image is a time-consuming process. To overcome this problem, many works have used the iterative search approaches to find the best focus point [5], [6]. A Fourier transform-based autofocusing method is also presented in [7]. In order to provide a dynamic autofocus, a reliable and accurate method has to be developed. In this paper, we present a real-time method where the best focus is achieved by controlling the focus step using an adaptive gain.

In the remainder of this paper, section II presents the experimental setup along with the details of focusing process in a SEM. Various sharpness functions are presented in section III. The proposed visual servoing-based autofocusing approach is presented in section IV. The experimental results are reported in section $\mathrm{V}$.

\section{EXPERIMENTAL SETUP}

The experimental setup architecture used for this work is shown in Fig. 1. It consists of a JEOL JSM 820 SEM along with two computers. The cross-section diagram of our SEM is shown in Fig. 2. Its electron column contains: an electron gun with tungsten filament, an anode, an objective aperture strip with 4 apertures of diameters 100, 70,50, and $30 \mu \mathrm{m}$, respectively, a secondary electron (SE) detector, two scan coils, an objective lens and a vacuum chamber equipped with a movable platform to place the sample. The primary computer (Intel Pentium 4, CPU $2.24 \mathrm{GHz}$ and $512 \mathrm{MB}$ of RAM) is solely responsible for SEM control and it is 


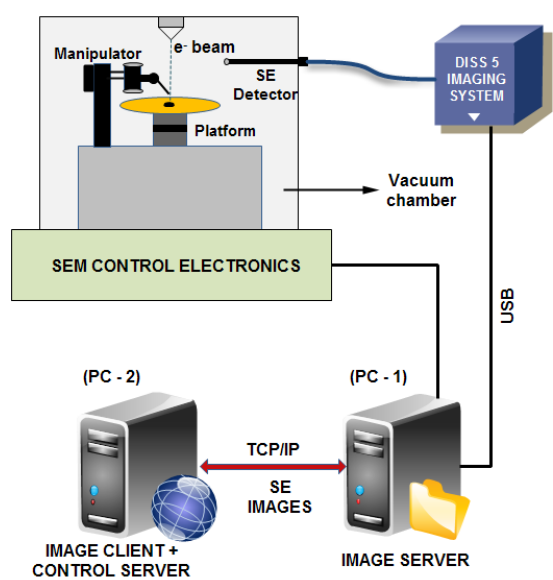

Fig. 1. Experimental setup architecture.

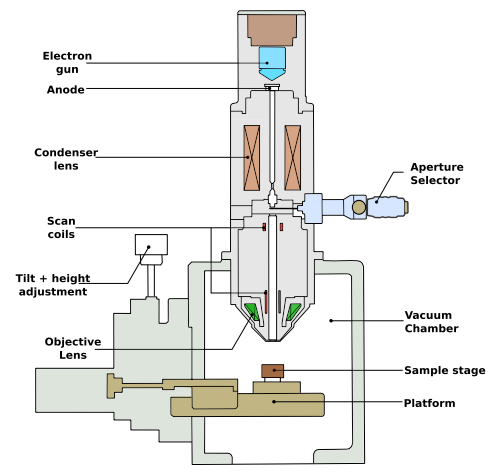

Fig. 2. Cross section diagram of JEOL JSM 820 SEM.

connected to the SEM electronics and an image acquisition system (DISS5 from Point Electronic $\mathrm{GmbH}$ ). The second PC (Intel Core 2 Duo, CPU $3.16 \mathrm{GHz}$, and $3.25 \mathrm{~GB}$ of RAM) is connected to the primary one using an Ethernet crossover cable. The communication between the two PCs is accomplished by implementing a client-server model using TCP/IP protocol, where the server program runs on the primary computer. The server is mainly responsible for setting the focus step and receiving images from the acquisition system. Image client receives these images from server and transmits them to the control server. Later, sharpness analysis and calculations are performed by the control server and a focus control command is issued to the image server via client. The possible magnification with the SEM ranges from $10 \times$ to $100,000 \times$ with an achievable resolution of $3 \mathrm{~nm}$.

\section{A. Focusing in SEM}

The two sets of electromagnetic lenses that contribute towards the focusing in SEM are condenser and objective lenses. The condenser lenses lying above the aperture strip are mainly responsible for controlling the spot size. Initially, these lenses converges the electron beam to a spot. Later, this beam flares out again and passes through the aperture, where low energy and non-directional electrons are filtered out. The objective lenses that are present below the aperture converges the beam once again and focus it onto the sample surface. Coarse focusing of the sample surface is performed by adjusting the electronic working distance that is attained by modifying the current passing through the objective

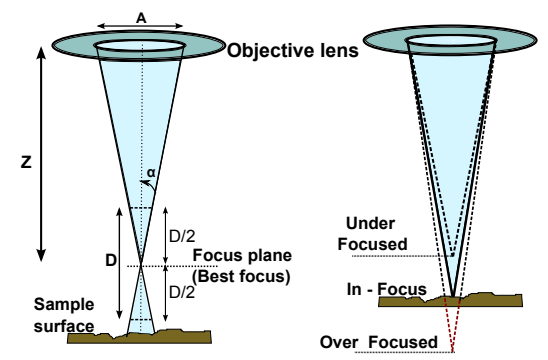

Fig. 3. (a) Depth of focus in SEM (b) Various focusing scenarios in SEM.

lenses. This distance is electronically measured between the focused plane (on the sample surface) and the lower pole piece of the objective lens (electron column) and is not to be confused with the sample stage height which can be controlled externally. Fig. 3(a) shows the focusing geometry in SEM. At a distance $D / 2$ on both sides of the focus plane for a selected magnification, the beam diameter is two times the pixel diameter and produces a blur image. Over the distance $D$ (depth of focus), the image appears to be acceptably in focus. The depth of focus D is given by (1).

$$
D=\frac{r Z}{A M}[\mu m]
$$

So, focus is mainly dependent on the size of the aperture $(A)$ and working distance $(Z)$ at any given magnification $(M)$ and resolution $(r)$. Fig. 3(b) reports the three main scenarios in the focusing procedure (i.e. focus, over focus and under focus).

The JEOL SEM is installed with a dynamic focusing module that modifies the current passing through the objective lens and thus the electronic working distance can be preselected. As stated earlier the DISS5 system has been used for this work, it provides a simple control for the focus by linking the electronic working distance with a series of focus steps (i.e. each focus step modifies the working distance to get a focused image). The relation between focus steps and electronic working distance is shown in Fig. 4. Subsequently, the value of working distance $(Z)$ for any given focus step $(F)$ can be computed by using the curve equation obtained by approximating it with a polynomial given in (2).

$$
Z= \begin{cases}50, & \text { if } F \leq 813 \\ 1, & \text { if } F \geq 1713 \\ p_{1} F^{4}+p_{2} F^{3}+p_{3} F^{2}+p_{4} F+p_{5}, & \text { otherwise }\end{cases}
$$

where, $p_{1} \ldots p_{5}$ are the polynomial coefficients.

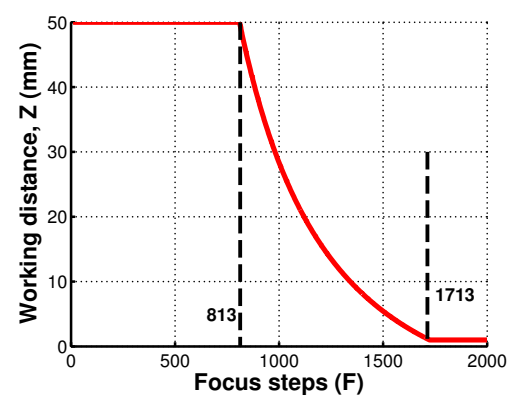

Fig. 4. Relationship between the focus step and working distance. 


\section{IMAGE SHARPNESS FUNCTIONS}

In the context of this work, various sharpness measures that have been used in many focusing algorithms are studied [4], [8] and [9]. The principle of accomplishing autofocusing based on the computed sharpness score using a sharpness function is shown in Fig. 5. It is considered that the image with best focus has the maximum value of sharpness score. Different sharpness functions are explained below using the following notations: sharpness function $\mathbf{S}$, image $\mathbf{I}_{M \times N}$ where $M$ and $N$ are the width and height, intensity $I(u, v)$ where $u$ and $v$ are pixel coordinates.

\section{A. Derivative-based sharpness functions}

Derivative-based sharpness functions are widely used in many experiments. The underlying principle behind these methods is that the focused images have high differences between the neighboring pixels than in out-of-focus images.

1) Laplacian: This function convolves the image with a Laplacian edge detection mask given by $L=[-1-4-$ $1 ;-420-4 ;-1-4-1]$ to compute the second derivative $d(u, v)$ of the image. The sharpness function is given by (3).

$$
\mathbf{S}_{L}=\sum_{M} \sum_{N}|d(u, v)|
$$

2) Brenner: Brenner's function uses the difference between a pixel value and its second neighboring pixel value.

$$
\mathbf{S}_{B}=\sum_{M} \sum_{N}|I(u, v)-I(u+2, v)|
$$

\section{B. Statistics-based sharpness functions}

Usually, the statistics-based measures are less sensitive to image noise and are widely used in many autofocusing algorithms.

1) Variance: This function computes the variations in the grey level intensities among the pixels.

$$
\mathbf{S}_{V}=\frac{1}{M N} \sum_{M} \sum_{N}(I(u, v)-\mu)^{2}
$$

where, $\mu$ is the image mean intensity and is given by (6).

$$
\mu=\frac{1}{M N} \sum_{M} \sum_{N} I(u, v)
$$

2) Normalized variance: This function normalizes the final value by the pixel mean intensity.

$$
\mathbf{S}_{N V}=\frac{1}{M N} \frac{1}{\mu} \sum_{M} \sum_{N}(I(u, v)-\mu)^{2}
$$

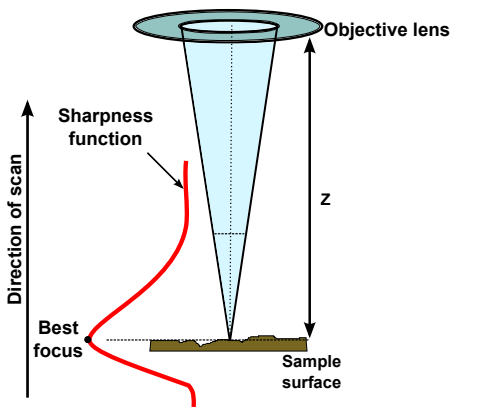

Fig. 5. Autofocusing principle in SEM.
3) Correlation: Two focusing measures using the correlation technique have been proposed. One is based on the autocorrelation function (ACF) and the other is based on standard deviation function. The ACF-based sharpness function is given by (8).

$$
\begin{gathered}
\mathbf{S}_{A C}=\sum_{u=1}^{M-1} \sum_{v=1}^{N} I(u, v) \cdot I(u+1, v)- \\
\sum_{u=1}^{M-2} \sum_{v=1}^{N}=I(u, v) \cdot I(u+2, v)
\end{gathered}
$$

The standard deviation-based function is given by (9).

$$
\mathbf{S}_{s t d}=\sum_{M} \sum_{N} I(u, v) \cdot I(u+1, v)-M \cdot N \cdot \mu^{2}
$$

\section{Wavelets-based sharpness functions}

Various sharpness functions have been proposed using the wavelet transform [10].

1) Wavelet-1: It applies both high-pass $(H)$ and lowpass $(L)$ filters to an image and divides it into four subimages $L L, L H, H L$ and $H H$. Focus score is computed by summing the absolute values in $H L, L H$ and $H H$ regions.

$$
\begin{gathered}
\mathbf{S}_{W 1}=\sum_{M} \sum_{N}\left|W_{H L}(u, v)\right|+\left|W_{L H}(u, v)\right|+ \\
\left|W_{H H}(u, v)\right|
\end{gathered}
$$

2) Wavelet-2: This function sums the variances in $H L$, $\mathrm{LH}$ and $\mathrm{HH}$ regions. The mean values in computing the variances for each region are computed using the absolute values.

$$
\begin{gathered}
\mathbf{S}_{W 2}=\frac{1}{M N} \sum_{M} \sum_{N}\left(\left|W_{H L}(u, v)\right|-\mu_{H L}\right)^{2}+ \\
\left(\left|W_{L H}(u, v)\right|-\mu_{L H}\right)^{2}+ \\
\left(\left|W_{H H}(u, v)\right|-\mu_{H H}\right)^{2}
\end{gathered}
$$

The evaluation results of the different sharpness functions are shown in Fig. 6. It is noticed that all the functions have achieved a maximum value at the same focus image with more or less efficiency. Out of all, the normalized variancebased method resulted in a curve with less noise and a welldefined peak in less time. Thus, in the design of the visual servoing-based autofocusing, we choose this method as our sharpness function for estimating the sharpness score.

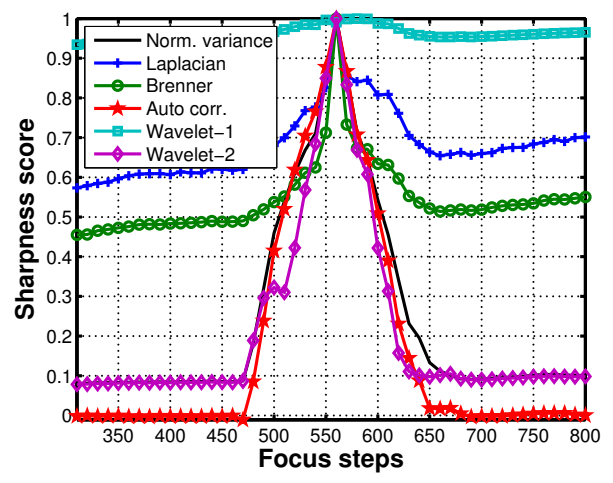

Fig. 6. Performance of various sharpness functions. 


\section{VISUAL SERVOING-BASED AUTOFOCUSING}

\section{A. Traditional visual servoing approach}

Visual servoing refers to the control of a robot's motion using the continuous image feedback information [11]. To achieve this, a set of visual features are derived from the images and are compared with the visual features from the reference image. In the basic approach of visual servoing, a control law is designed such that the robot will move to a desired pose $\mathbf{p}^{*}$, having $\mathbf{s}^{*}$ visual features, using the computed visual features $\mathbf{s}$, at the current pose $\mathbf{p}$. The main goal lies in minimizing the error function, e given by (12).

$$
\mathbf{e}=\mathbf{s}-\mathbf{s}^{*}
$$

For designing the vision-based control scheme, an interaction matrix $\mathbf{L}$ that links the time variation of the current visual features $\dot{\mathbf{s}}$ with the camera instantaneous velocity $\mathbf{v}_{\mathbf{c}}$ is required. The relationship is then given by $\dot{\mathbf{s}}=\mathbf{L v}_{\mathbf{c}}$. Using $\mathbf{e}$ and $\dot{\mathbf{s}}$, the camera velocity is given by (13).

$$
\mathbf{v}_{\mathbf{c}}=-\lambda \mathbf{L}^{\dagger} \dot{\mathbf{e}}
$$

where, $\lambda$ is the gain to assure an exponential decrease in the error, $\mathbf{L}^{\dagger}$ is the pseudo-inverse of the $\mathbf{L}$ and $\dot{\mathbf{e}}$ is the time variation of $\mathbf{e}$. For a $2 \mathrm{D}$ point feature $m(u, v)$ in the image frame that is a projection of the $3 \mathrm{D}$ point $M(X, Y, Z)$ in the camera frame, the interaction matrix $\mathbf{L}$ associated with $m$ is given by (14).

$$
\mathbf{L}=\left[\begin{array}{cccccc}
\frac{-1}{Z} & 0 & \frac{u}{Z} & u v & -(1+u)^{2} & v \\
0 & \frac{-1}{Z} & \frac{v}{Z} & -(1+v)^{2} & -u v & -u
\end{array}\right]
$$

\section{B. Autofocusing using visual servoing}

This work has been performed utilizing the idea of basic visual servoing technique. Instead of minimizing the error function, the proposed method maximizes the normalized variance sharpness function given by (7). Rather than computing the local visual features, the global image information is used in this work. This type of visual servoing is called as direct visual servoing [12]. Most importantly, no reference image or features are used; instead, the method converges to a best focus position i.e. when the sharpness function reaches its maximum value.

If our goal is to maximize the sharpness function, it is first required to observe the variation of its behavior with respect to the focus steps i.e. with the change in electronic working distance. Fig. 7(a) shows this behavior of the sharpness function at two different magnifications. It is then observed that, for a specific range of working distance (inside the peak) we get images with some details and the rest are dark or bright images with varying noise. From this, the primary goal is to drive the control to reach the focus range. Keeping this in mind, the initial control law has been designed using an adaptive gain that changes with the sharpness score. The corresponding vision-based objective function $(\mathbf{C})$ is given by (15).

$$
\mathbf{C}=\lambda\left(\frac{\nabla \mathbf{S}}{\|\nabla \mathbf{S}\|}\right)
$$

where, $\lambda$ is the adaptive gain given by (16).

$$
\lambda= \begin{cases}\alpha\left(\frac{\mathbf{S}_{0}}{\mathbf{S}}\right)^{2}, & \text { if } \frac{\mathbf{S}_{0}}{\mathbf{S}}<1, \\ \alpha, & \text { elsewhere. }\end{cases}
$$

where, $\alpha$ is a positive integer, $\mathbf{S}_{0}$ and $\mathbf{S}$ are the sharpness scores computed from first image and the current image. The unit vector $\left(\frac{\nabla \mathbf{S}}{\|\nabla \mathbf{S}\|}\right)$ provides the driving direction. Using (16), the primary task function $\mathbf{T}_{1}$ now takes the form given in (17).

$$
\mathbf{T}_{1}=F_{c}+\mathbf{C}
$$

where, $F_{c}$ is the current focus step. The underlying idea is to have a maximum amount of gain at the region far from the focus. This ensures that the focus step is big enough to reach the focus region. Next, to have a minimum amount of gain at the region near focus such that the focus step is small. As the primary task can only drive the focus step using sharpness information, it does not have any direct linking with the working distance. In visual servoing, if the primary task does not constrain all degrees of freedom, a secondary task can be added as it has no effect on the primary task [13]. So, for this problem of autofocusing using visual servoing, a secondary task $\left(\mathbf{T}_{2}\right)$ has been realized, that links the electronic working distance $(Z)$ with the gradient of sharpness function. Later, it has been used as a stopping criterion for the overall process. The secondary task function is given by (18).

$$
\mathbf{T}_{2}=\left(\frac{\partial \mathbf{S}}{\partial Z}\right) \mathbf{L}_{z}
$$

where, $\left(\frac{\partial \mathbf{S}}{\partial Z}\right)$ is the gradient of the sharpness function with respect to the working distance $Z$ and $\mathbf{L}_{z}$ is the interaction matrix that links the gradient with the working distance.

Here, assuming the change in the $Z$ is small (especially near best focus point), the gradient has been treated as the difference between consecutive sharpness scores given by (19). The interaction matrix $\mathbf{L}_{z}=\frac{-1}{Z}$ where, the $Z$ value at a specific focus step is obtained from (2).

$$
\frac{\partial \mathbf{S}}{\partial Z}=\frac{\mathbf{S}_{i}-\mathbf{S}_{i-1}}{\triangle Z}
$$

Using (17) and (18), the final control law to control the focus step is given by (20).

$$
F=F_{c}+\underbrace{\lambda\left(\frac{\nabla \mathbf{S}}{\|\nabla \mathbf{S}\|}\right)}_{\text {main focusing task }}+\overbrace{\left(\frac{\partial \mathbf{S}}{\partial Z}\right) \mathbf{L}_{z}}^{\text {secondary task }}
$$

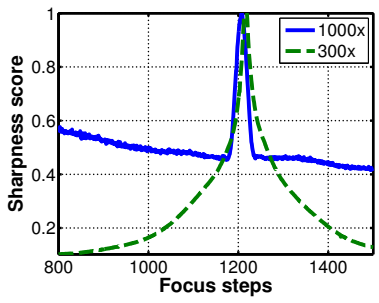

(a)

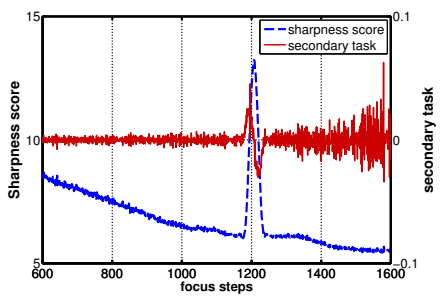

(b)
Fig. 7. (a) Sharpness score variation with respect to focus steps at $300 \times$ and $1000 \times$ magnifications (b) secondary task variation with respect to the focus steps. 
The relationship between the sharpness score and the secondary task functions at $1000 \times$ magnification is depicted in Fig. 7(b). The secondary task function thus provides the derivative information of the sharpness score. When the sharpness function reaches it maximum, the secondary task function crosses zero and hence the overall process can be stopped at this point. Because of the presence of noise in the images, the secondary task function have some peaks near zero, to avoid this effect a value of -0.001 has been treated as the stopping limit in this work.

\section{EXPERIMENTS WITH THE SYSTEM}

This work has been performed to investigate and find a fast and reliable autofocusing method for SEM. Many experiments are performed to evaluate the proposed approach of autofocusing based on the visual servoing technique. The sample used for imaging is a microscale calibration template containing chessboard patterns that is fabricated at FEMTO$\mathrm{ST}$ institute. The acceleration voltage used to produce the beam is $15 \mathrm{kV}$. All experiments are carried out using the secondary electron images with a size of $512 \times 512$ pixels.

\section{A. Evaluation of the method}

Initial experiments have been performed to validate the proposed method at different magnifications. The tests are conducted using an aperture size of $50 \mu \mathrm{m}$ and $\alpha=400$ at $300 \times$ and $1000 \times$ magnifications. The brightness and contrast levels are set to the optimal values. The dwell time i.e. the time to scan one pixel is set to $360 \mathrm{~ns}$ to achieve an acquisition speed of 2.1 frames per second. Fig. 8(a) and Fig. 8(b) show the sharpness scores given by (7) and the corresponding gain values at $300 \times$ and $1000 \times$ magnifications respectively. Fig. 9 shows the secondary task function at $300 \times$ and $1000 \times$ magnifications. From the obtained results, it can be noticed that the focusing process stopped precisely at the maximum of sharpness function i.e. at this point the secondary task function reached -0.001 .

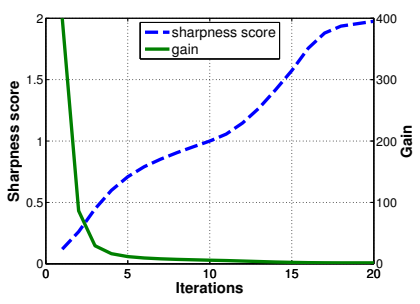

(a)

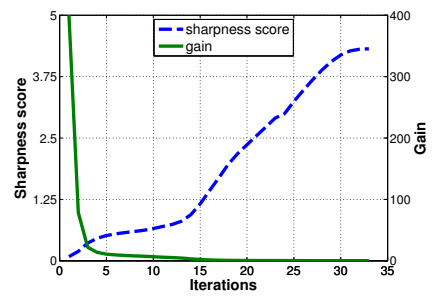

(b)
Fig. 8. Sharpness score and corresponding gain values at (a) $300 \times$ (b) $1000 \times$ magnifications respectively.

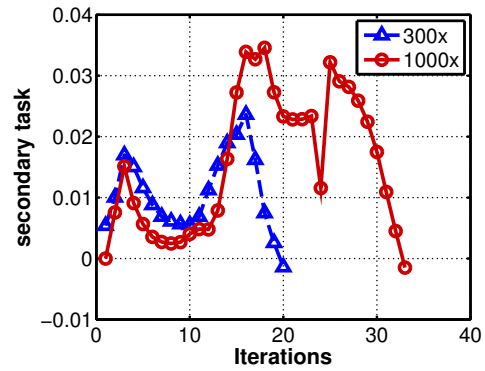

Fig. 9. Secondary task function at $300 \times$ and $1000 \times$ magnifications.

\section{B. Speed and accuracy test}

The performance of the proposed autofocusing method is evaluated by comparing with the traditional search-based algorithms at different experimental conditions. An iterative search approach has been employed for this purpose. The search-based method is implemented over the total range of focus steps using three iterations. For each iteration, the total range of focus steps is divided into four subregions and searched for the global maxima with a fixed step size. The region containing the maximum value is then used for the next iteration. The step sizes used for each iteration are 50, 20 and 1 , respectively. The maximum value found after the final iteration is the best focus point. The accuracy obtained by this method has been treated as the best accuracy, since it scans each step for the best focus in its final iteration. Hence, the focus step returned by this method is used as the reference step for computing the step error.

The proposed method is compared to the iterative searchbased approach in terms of total time taken and accuracy of focusing at different magnifications. Here, the total time taken also includes the time taken to acquire and transfer the images. The obtained results using optimal values of brightness, contrast and scan speed are summarized in Table I. Table II shows the results obtained with an increase in brightness. Table III summarizes the results achieved with a changed dwell time of $180 \mathrm{~ns}$. This change in the dwell time increases the noise levels in the images. Table IV shows the results obtained using a silicon dioxide sample containing the microparts. The main reason behind this test is that the surface of this sample is not a good conductor. Due to this, charges are accumulated on the sample surface resulting in the images with grey level fluctuations. All these tests are performed using $\alpha=300$. Finally, Table V summarizes various speeds that can be achieved with proposed visual servoing-based method by changing the $\alpha$ value. Fig. 10 shows some of the screenshots captured during various tests. The obtained results show the repeatability, robustness and speed of the proposed method regardless of the operating conditions.

TABLE I

TIME TAKEN AND ACCURACY AT OPTIMAL CONDITIONS.

\begin{tabular}{|c|c|c|c|c|c|}
\hline \multirow{2}{*}{ Mag $(\times)$} & \multicolumn{2}{|c|}{ Time $(s)$} & \multicolumn{3}{c|}{$\begin{array}{c}\text { Accuracy (Focus steps) } \\
\text { (Focus step } \simeq 0.3 \mathrm{~mm} \text { ) }\end{array}$} \\
\cline { 2 - 6 } & proposed & search & proposed & search & step error \\
\hline 300 & 42.703 & 117.97 & 1222 & 1219 & 3 \\
\hline 500 & 48.5 & 109.62 & 1220 & 1218 & 2 \\
\hline 800 & 43.46 & 112.03 & 1221 & 1219 & 2 \\
\hline 1000 & 48.42 & 109.4 & 1222 & 1219 & 3 \\
\hline
\end{tabular}

TABLE II

TIME TAKEN AND ACCURACY AT CHANGED BRIGHTNESS.

\begin{tabular}{|c|c|c|c|c|c|}
\hline \multirow{2}{*}{ Mag $(\times)$} & \multicolumn{2}{|c|}{ Time $(s)$} & \multicolumn{3}{c|}{$\begin{array}{c}\text { Accuracy (Focus steps) } \\
\text { (Focus step } \simeq 0.3 \mathrm{~mm} \text { ) }\end{array}$} \\
\cline { 2 - 6 } & proposed & search & proposed & search & step error \\
\hline 300 & 42.06 & 110.64 & 1217 & 1213 & 4 \\
\hline 500 & 49.48 & 118.06 & 1218 & 1213 & 5 \\
\hline 800 & 48.29 & 111.6 & 1215 & 1212 & 3 \\
\hline 1000 & 51.37 & 111.74 & 1216 & 1213 & 3 \\
\hline
\end{tabular}


TABLE III

TIME TAKEN AND ACCURACY WITH CHANGE IN THE DWELL TIME.

\begin{tabular}{|c|c|c|c|c|c|}
\hline \multirow{2}{*}{ Mag $(\times)$} & \multicolumn{2}{|c|}{ Time $(s)$} & \multicolumn{3}{c|}{$\begin{array}{r}\text { Accuracy (Focus steps) } \\
\text { (Focus step } \simeq 0.3 \mathrm{~mm} \text { ) }\end{array}$} \\
\cline { 2 - 6 } & proposed & search & proposed & search & step error \\
\hline 300 & 17.39 & 64.28 & 1224 & 1219 & 5 \\
\hline 500 & 16.23 & 62.15 & 1223 & 1219 & 4 \\
\hline 800 & 16.61 & 62.84 & 1222 & 1219 & 3 \\
\hline 1000 & 14.37 & 65.09 & 1222 & 1218 & 4 \\
\hline
\end{tabular}

TABLE IV

TIME TAKEN AND ACCURACY WITH SILICON DIOXIDE SAMPLE AT OPTIMAL CONDITIONS.

\begin{tabular}{|c|c|c|c|c|c|}
\hline \multirow{2}{*}{ Mag $(\times)$} & \multicolumn{2}{|c|}{ Time $(s)$} & \multicolumn{3}{c|}{$\begin{array}{c}\text { Accuracy (Focus steps) } \\
\text { (Focus step } \simeq 0.3 \mathrm{~mm} \text { ) }\end{array}$} \\
\cline { 2 - 6 } & proposed & search & proposed & search & step error \\
\hline 300 & 39.1 & 116.82 & 1473 & 1471 & 2 \\
\hline 500 & 36.45 & 99.07 & 1468 & 1471 & -3 \\
\hline 800 & 41.61 & 108.65 & 1472 & 1471 & 1 \\
\hline 1000 & 34.07 & 101.4 & 1476 & 1473 & 3 \\
\hline
\end{tabular}

\section{CONCLUSION}

In this paper, new, fast and robust visual servoing-based autofocusing method for SEM has been presented. Unlike the traditional methods, the developed method performs the focusing by controlling the size of the focus step using an adaptive gain. The secondary task used in the control law precisely stops the overall process, when a best focus position is reached. For this work, a normalized variance sharpness function has been selected to produce the sharpness score of the images. Based on the sharpness score, the value of the gain is adjusted such that a best focus position is ensured. Obtained experimental results prove the fastness of the developed method over existing search-based approaches and precision in finding the best focus. The proposed autofocusing approach has also been validated at different experimental conditions and the obtained results prove the robustness of the method. Future work will be devoted to use this technique in performing the autonomous nanoparts handling application using SEM.

\section{ACKNOWLEDGMENT}

Authors would like to thank Mr. Joel Agnus of AS2M, FEMTO-ST for his support in fixing the problems with SEM.

\section{REFERENCES}

[1] T. Wich, T. Sievers, M. Jahnisch, H. Hulsen, and S. Fatikow, "Nanohandling automation within a scanning electron microscope," in Industrial Electronics, 2005. ISIE 2005. Proceedings of the IEEE International Symposium on, vol. 3, June, pp. 1073-1078 vol. 3.

[2] C. in Colour. (2013) Understanding camera autofocus. [Online]. Available: http://www.cambridgeincolour.com/tutorials/ camera-autofocus.htm

[3] F. Groen, I. Young, and G. Ligthart, "A comparison of different focus functions for use in autofocus algorithms," Cytometry, vol. 6, no. 2, pp. 81-91, 2005.

[4] Y. Sun, S. Duthaler, and B. Nelson, "Autofocusing algorithm selection in computer microscopy," in IEEE/RSJ International Conference on Intelligent Robots and Systems.(IROS 2005). IEEE, 2005, pp. 70-76.

[5] C. Batten, "Autofocusing and astigmatism correction in the scanning electron microscope," Master's thesis, University of Cambridge, 2000.

[6] M. Rudnaya, R. Mattheij, and J. Maubach, "Iterative autofocus algorithms for scanning electron microscopy," Microscopy and Microanalysis, vol. 15, no. S2, pp. 1108-1109, 2009.
TABLE V

TIME TAKEN BY THE PROPOSED METHOD WITH VARIOUS $\alpha$ VALUES

\begin{tabular}{|c|c|c|c|c|}
\hline Mag $(\times)$ & $\alpha=100$ & $\alpha=200$ & $\alpha=300$ & $\alpha=400$ \\
\hline 300 & 96.01 & 69.7 & 42.91 & 19.7 \\
\hline 1000 & 98.3 & 70.17 & 48.4 & 21.4 \\
\hline
\end{tabular}

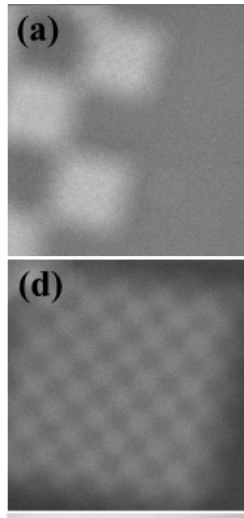

(g)
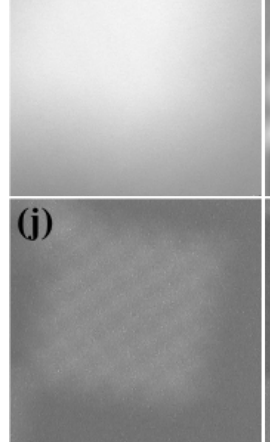

(m)

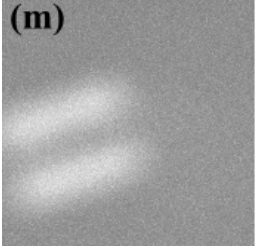

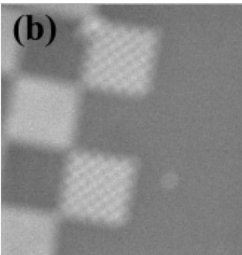
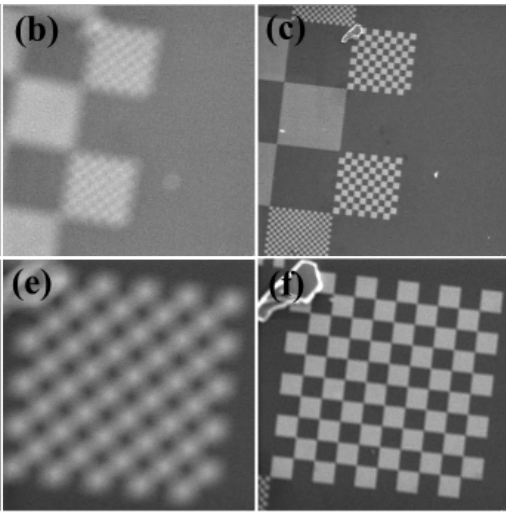

(h)
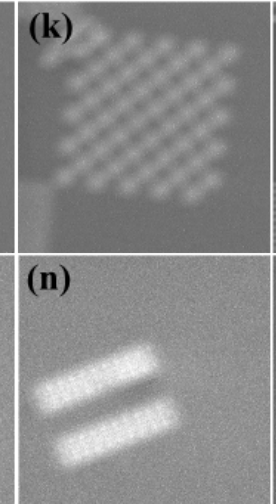
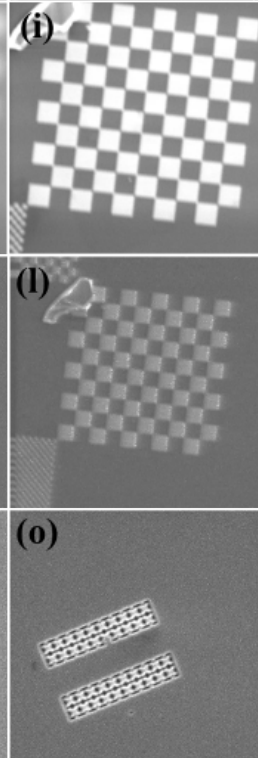

Fig. 10. Screenshots of the autofocusing process during different tests. Last column show sharp images. (a) to (c) at $300 \times$ magnifications (d) to (f) at $1000 \times$ magnifications $(\mathrm{g})$ to (i) change in brightness at $1000 \times$ magnifications (j) to (l) using a dwell time of $180 \mathrm{~ns}$. (m) to (n) silicon sample containing the microparts at low magnification.

[7] K. Ong, J. Phang, and J. Thong, "A robust focusing and astigmatism correction method for the scanning electron microscope," Scanning, vol. 19 , no. 8, pp. 553-563, 1997.

[8] M. Rudnaya, R. Mattheij, and J. Maubach, "Evaluating sharpness functions for automated scanning electron microscopy," Journal of microscopy, vol. 240, no. 1, pp. 38-49, 2010.

[9] M. Subbarao, T. Choi, and A. Nikzad, "Focusing techniques," Optical Engineering, vol. 32, no. 11, pp. 2824-2836, 1993.

[10] G. Yang and B. Nelson, "Wavelet-based autofocusing and unsupervised segmentation of microscopic images," in IEEE/RSJ International Conference on Intelligent Robots and Systems. (IROS 2003)., vol. 3, oct. 2003 , pp. $2143-2148$ vol.3.

[11] F. Chaumette and S. Hutchinson, "Visual servo control. I. basic approaches," Robotics \& Automation Magazine, IEEE, vol. 13, no. 4, pp. 82-90, 2006.

[12] B. Tamadazte, N. Le-Fort Piat, and E. Marchand, "A direct visual servoing scheme for automatic nanopositioning," IEEE/ASME Transactions on Mechatronics, vol. 17, no. 4, pp. 728 -736, aug. 2012.

[13] E. Marchand, "Control camera and light source positions using image gradient information," in IEEE International Conference on Robotics and Automation. (ICRA 2007). IEEE, 2007, pp. 417-422. 